\title{
INTEGRAL spectroscopy of the accreting millisecond pulsar XTE J1807-294 in outburst
}

\author{
M. Falanga ${ }^{1}$, J. M. Bonnet-Bidaud ${ }^{1}$, J. Poutanen ${ }^{2}$, R. Farinelli ${ }^{3}$, A. Martocchia ${ }^{4}$, P. Goldoni ${ }^{1}$, J. L. Qu $^{5}$, \\ L. Kuiper ${ }^{6}$, and A. Goldwurm ${ }^{1}$ \\ ${ }^{1}$ CEA Saclay, DSM/DAPNIA/Service d'Astrophysique (CNRS FRE 2591), 91191 Gif-sur-Yvette, France \\ e-mail: mfalanga@cea.fr \\ 2 Astronomy Division, PO Box 3000, 90014 University of Oulu, Finland \\ 3 Dipartimento di Fisica, Università di Ferrara, via Paradiso 12, 44100 Ferrara, Italy \\ 4 Observatoire Astronomique, 11 rue de l'Université, 67000 Strasbourg, France \\ 5 Laboratory for Particle Astrophysics, Institute of High Energy Physics, CAS, Beijing 100039, PR China \\ ${ }^{6}$ SRON National Institute for Space Research, Sorbonnelaan 2, 3584 CA Utrecht, The Netherlands
}

Received 20 December 2004 / Accepted 7 March 2005

\begin{abstract}
The transient X-ray accreting millisecond pulsar XTE J1807-294 was observed during its February/March 2003 outburst by INTEGRAL, partly simultaneously with the XMM-Newton and RXTE satellites. We present here the first study of the 0.5-200 keV broad-band spectra of the source. On February 28, the source spectrum was consistent with thermal Comptonization by electrons of temperature $\sim 40 \mathrm{keV}$, considerably higher than the value $(\sim 10 \mathrm{keV})$ previously derived from the low energy XMM-Newton data alone. The source is detected by INTEGRAL up to $200 \mathrm{keV}$ with a luminosity in the energy band $(0.1-200) \mathrm{keV}$ of $1.3 \times 10^{37} \mathrm{erg} \mathrm{s}^{-1}$ (assuming a distance of $8 \mathrm{kpc}$ ). 22 days later the luminosity dropped to $3.6 \times 10^{36} \mathrm{erg} \mathrm{s}^{-1}$. A re-analysis of XMM-Newton data yields the orbital Doppler variations of the pulse period and refines the previous ephemeris. For this source, with shortest orbital period of any known binary radio or X-ray millisecond pulsar, we constrain the companion mass $M_{\mathrm{c}}<0.022 M_{\odot}$, assuming minimum mass transfer driven by gravitational radiation. Only evolved dwarfs with a $\mathrm{C} / \mathrm{O}$ composition are consistent with the Roche lobe and gravitational radiation constraints, while He dwarfs require an unlikely low inclination.
\end{abstract}

Key words. accretion, accretion disks - X-rays: binaries - stars: individual: XTE J1807-294 - stars: neutron

\section{Introduction}

Neutron stars (NS) in low-mass X-ray binaries (LMXB) are spun up by accretion to millisecond periods. The end point of their evolution is expected to be a millisecond pulsar (MSP), i.e. a rapidly spinning NS with a rather weak, $\sim 10^{8} \mathrm{G}$, surface magnetic field (for a review see Bhattacharya \& van den Heuvel 1991). Although evidence for rapidly spinning neutron stars in LMXBs was obtained from the burst oscillations that were seen during type I X-ray bursts in several systems (see Strohmayer 2001) and from the discovery of kHz QPOs (see van der Klis 2000, for a review), the detection of millisecond pulsations in the persistent emission remained elusive for many years, until the discovery of the first accreting millisecond pulsar by Wijnands \& van der Klis (1998). Since that time, a total of six accreting MSPs have been detected (see review by Wijnands 2005). The latest one, IGR J00291+5934, was discovered very recently with INTEGRAL (Eckert et al. 2004) and turned out to be the fastest rotating object among the six with a frequency of $599 \mathrm{~Hz}$ (Markwardt et al. 2004). The other pulsar spin frequencies are between 180 and $435 \mathrm{~Hz}$. All of the accreting MSPs are X-ray transients. The accreting MSPs spend most of the time in a quiescent state, with X-ray luminosities of the order of $10^{31}-10^{32} \mathrm{erg} \mathrm{s}^{-1}$ and sometimes go into outburst reaching luminosities of $\leq 10^{38} \mathrm{erg} \mathrm{s}^{-1}$. The millisecond pulsar XTE J1807-294 was discovered in outburst on February 2003 with the Rossi X-ray Timing Explorer (RXTE) during the monitoring observations of the Galactic center region (Markwardt et al. 2003). It has a pulse period of $5.25 \mathrm{~ms}$. The orbital period of $\sim 40 \mathrm{~min}$ is the shortest one of any known binary radio or X-ray millisecond pulsar. An XMM-Newton Target of Opportunity observation was used to derive the low energy spectrum and the source pulse profile (Campana et al. 2003; Kirsch et al. 2004). In this paper, we analyze the INTEGRAL hard X-ray spectra of this source obtained simultaneously with XMM-Newton and RXTE during the February/March 2003 outburst.

\section{Observations and data analysis}

\subsection{INTEGRAL}

The pulsar was observed by INTEGRAL (Winkler et al. 2003) twice. The first observation was obtained during a Target of 
Opportunity (ToO) pointing at the Galactic center region, performed from February 28 to March 1, 2003 (satellite revolution 46) which was contemporaneous with the RXTE observation of the pulsar. The second observation during a Galactic Center Deep Exposure (GCDE) observation was contemporaneous with both the XMM-Newton and RXTE observations on March 20-22, 2003 (satellite revolutions 52/53). Hereafter we call the two datasets rev-46 and rev-52, respectively.

We observed XTE J1807-294 with one of the main gamma-ray instruments, IBIS/ISGRI (Ubertini et al. 2003; Lebrun et al. 2003), on-board the spacecraft. The effective exposure time was $157 \mathrm{ks}$ and $263 \mathrm{ks}$ for rev-46 and rev-52, respectively. The data were extracted for all pointings with a source position offset $\leq 12^{\circ}$. Data reduction was performed using the standard Offline Science Analysis (OSA) version 3.0 distributed by the INTEGRAL Science Data Center (Courvoisier et al. 2003). The algorithms used in the analysis are described in Goldwurm et al. (2003).

\subsection{XMM-Newton}

XTE J1807-294 was observed with XMM-Newton (Jansen et al. 2001) on March 22, 2003 for about $17 \mathrm{ks}$ with the first results reported in Campana et al. (2003) and Kirsch et al. (2004). The data, simultaneous with the rev-52 INTEGRAL dataset, were retrieved from the XMM-Newton database and re-analyzed to produce a $0.5-10 \mathrm{keV}$ spectrum contemporary with the INTEGRAL observations. We used the data from the EPIC-pn camera (Strüder et al. 2001) operating in Timing mode, the MOS1 camera operating in Small Window mode and MOS2 in Prime Full Window mode (Turner et al. 2001). The total amount of good exposure time selected was $14 \mathrm{ks}$ and $9 \mathrm{ks}$ for MOS2 and the other instruments, respectively. The Observation Data Files (ODFs) were processed to produce calibrated event lists using the XMM-Newton Science Analysis System (SAS version 5.4.1). The XMM-Newton data reduction and analysis was similar to the procedure described in Campana et al. (2003) with a special care taken to select the events and eliminate pile-up.

\subsection{RXTE}

We used publicly available data from the proportional counter array (PCA) and the High Energy X-ray Timing Experiment (HEXTE) on board RXTE. XTE J1807-294 was monitored from February 28 to June 16, 2003. We analyzed only the data contemporary with INTEGRAL for February 28 to March 1 and also with XMM-Newton on March 20-22. The effective exposure time for the first observation was $15.2 \mathrm{ks}$ and for the later observation $23 \mathrm{ks}$. We carried out a spectral analysis, using standard-2 data (with $16 \mathrm{~s}$ time resolution) for the PCA and standard cluster-A data for HEXTE. To improve the signal-tonoise for the PCA we extracted only the data from the top layers of PCU2 and PCU3. The data were extracted for the Good Time Intervals defined by standard criteria. The PCA response matrix was created by FTOOLS version 5.3 for 129 energy channels to cover the energy range from 2-60 keV. For HEXTE we

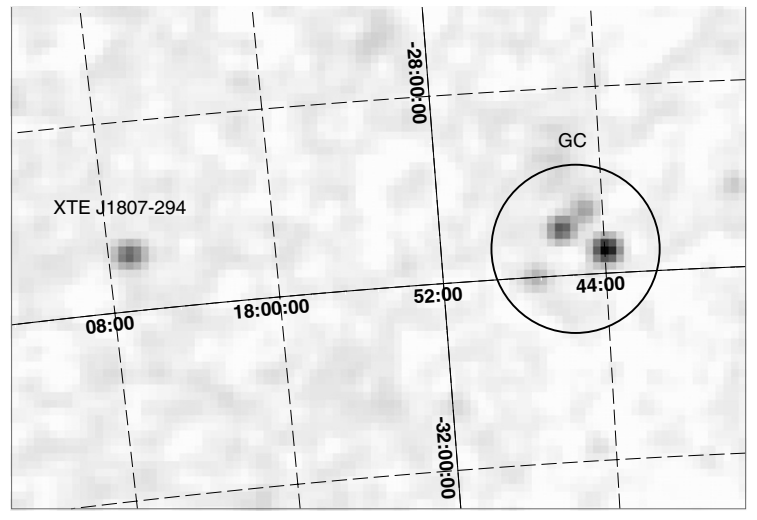

Fig. 1. The INTEGRAL IBIS/ISGRI mosaiced and deconvolved image in the $20-40 \mathrm{keV}$ energy interval from the rev- 46 observation. The image size is $\sim 9.5 \times 5^{\circ} .5$. The pixel size is $5^{\prime}$. The source XTE J1807-294 was detected at a significance of $28 \sigma$. The other sources in the Galactic center region (circle labeled GC) are 1E 1740.7-2942

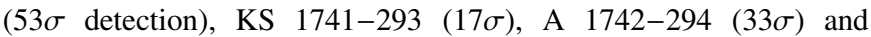
SLX 1744-300 $(14 \sigma)$.

used the standard 64 energy channel response matrix for the $15-250 \mathrm{keV}$ energy range.

\section{Results}

\subsection{IBIS/ISGRI image and light curves}

Figure 1 shows a significance map of the Galactic center region in the $20-40 \mathrm{keV}$ energy range. Single pointings were deconvolved and analyzed separately, and then combined in mosaic images. In the FOV of the image, XTE J1807-294 is clearly detected at a significance level of $28 \sigma$. In the $40-80 \mathrm{keV}$ energy band the source was detected at $14.5 \sigma$, and at higher energies, 80-120 keV, at $6 \sigma$ and from $120-200 \mathrm{keV}$ the detection significance dropped below $\sim 3 \sigma$. The source was observed at the position $\alpha_{\mathrm{J} 2000}=18^{\mathrm{h}} 07^{\mathrm{m}} 00^{\mathrm{s}} 38$ and $\delta_{\mathrm{J} 2000}=$ $-29^{\circ} 24^{\prime} 30^{\prime} .8$, with a positional error of $1^{\prime}$ at $90 \%$ confidence (Gros et al. 2003). This is fully consistent with the position given by Markwardt et al. (2003). It is located 5.7 away from the Galactic center.

The INTEGRAL 20-100 keV high-energy light curve has been extracted from the images using all available pointings (all GCDE observation with the source in the FOV from) and is shown in Fig. 2, averaged over 1-day intervals. A clear exponential decay $\mathrm{e}^{-t / \tau}$ with a time-scale of $\tau=19 \pm 2$ days is seen over the $\sim 60$ days of the INTEGRAL observations. The corresponding $R X T E / \mathrm{PCA}$ outburst light curve in the $2-20 \mathrm{keV}$ range is also shown in Fig. 2. The first five points are taken from Markwardt et al. (2003), where the source was observed with a peak 2-20 keV flux of $\sim 2 \times 10^{-9} \mathrm{erg} \mathrm{s}^{-1} \mathrm{~cm}^{-2}$. The light curve shows also some short flares during the decay around March 9, 15, 30 and April 5 (see Zhang et al. 2005). The RXTE decay time-scale, $\tau=19.22 \pm 0.04 \mathrm{~d}$, is fully consistent with the INTEGRAL value at higher energy. The equality of the RXTE and INTEGRAL timescales shows that the spectrum did not change much during the outburst. After 50 days the decay became slower with a time-scale of $122 \pm 3$ days. The 


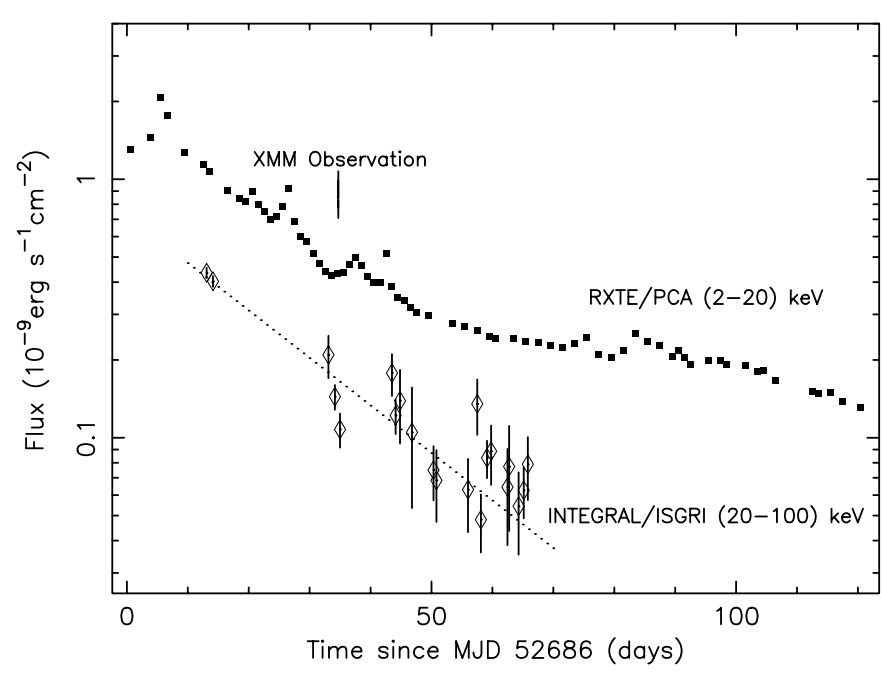

Fig. 2. RXTE/PCA and INTEGRAL/ISGRI light curves in the 2-20 keV and 20-100 keV energy band, respectively (averaged over 1-day intervals). The INTEGRAL data are taken from the GCDE observations and have been converted to flux units assuming a Comptonization model (see Table 1). The arrow indicates the $X M M$-Newton observation time. The dotted line corresponds to $F \propto$ $\mathrm{e}^{-t / 19.2 \mathrm{~d}}$ for the ISGRI data.

XTE J1807-294 outburst lasted much longer than that for other accretion powered MSPs such as SAX J1808.4-3658 (with $\tau \sim 14 \mathrm{~d}$ ) and XTE J1751-305 (with $\tau \sim 7 \mathrm{~d}$ ). XTE J1807-294 is up to now the only MSP which shows a decay of the outburst profile without a distinct knee as seen in the other five MSPs (see Wijnands 2005).

Using the rev-46 dataset with the highest $\mathrm{S} / \mathrm{N}$, detailed light curves in different energy bands have been produced, which are shown in Fig. 3. The count rates, averaged over $\sim 2.2 \mathrm{ks}$ intervals, have been converted to flux values using a Comptonization model (see Sect. 3.2). The hardness ratio as a function of time indicates that no significant spectral variability was detected. The hardness ratio between the highest energy band and the lowest energy band was found to be consistent with constant $0.5 \pm 0.1$. Pulsations with a period of $5.25 \mathrm{~ms}$ were also marginally detected in the the $20-50 \mathrm{keV}$ IBIS ISGRI energy band. The pulse fraction was $\sim 8 \%$. These results will be reported in Finger et al. (2005).

\subsection{Spectral analysis}

The spectral analysis was done using XSPEC version 11.3 (Arnaud 1996), combining INTEGRAL/ISGRI data with available simultaneous lower energy RXTE and XMM-Newton data. A constant factor was included in the fit to take into account the uncertainty in the cross-calibration of the instruments. A systematic error of $2 \%$ was applied to IBIS/ISGRI spectra which corresponds to the current uncertainty in the response matrix and $1 \%$ for all the XMM-Newton and RXTE instruments. All spectral uncertainties in the results are given at a $90 \%$ confidence level for a single parameter $\left(\Delta \chi^{2}=2.71\right)$. The distance, $D$, to the source is not known. As it might be related to the Galactic center, it is not unreasonable to assume $D \sim 8 \mathrm{kpc}$.

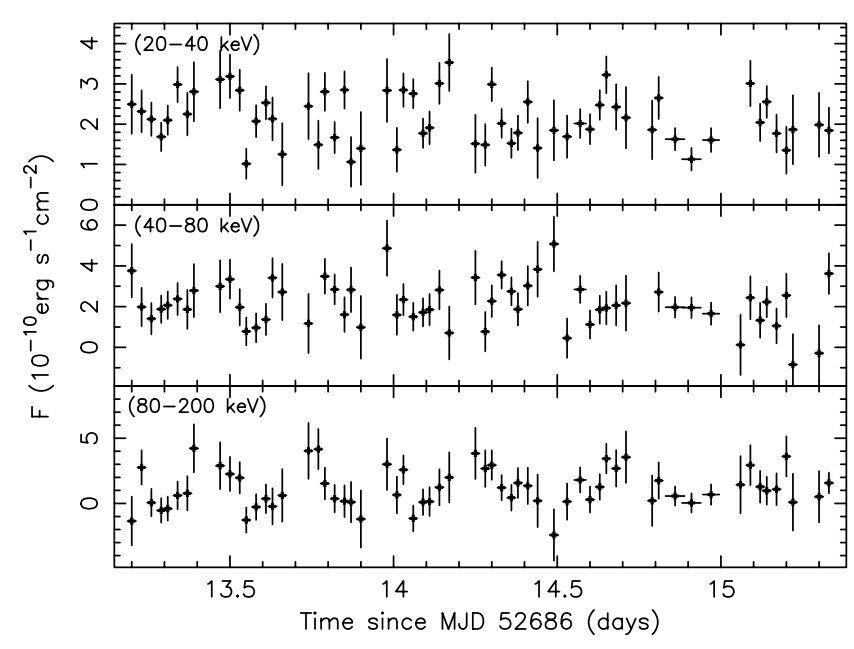

Fig. 3. The rev-46 high-energy light curve of XTE J1807-294 in different energy bands. Each point corresponds to a $\sim 2.2 \mathrm{ks}$ interval.

\subsubsection{The rev- 46 spectrum}

In the rev-46 dataset, the joint INTEGRAL/RXTE (2.5-200 keV) spectrum was first fitted with a simple model consisting of a photoelectrically-absorbed power-law (PL). Given that we were not able to constrain the $N_{\mathrm{H}}$ value (as the RXTE/PCA bandpass starts above $2.5 \mathrm{keV}$ ) we fixed it to the value found from XMM-Newton lower energy data (see Sect. 3.2.2 below). A simple PL model is found inadequate with $\chi^{2} /$ d.o.f. $=273 / 65$. The addition of a cut-off above $\sim 60 \mathrm{keV}$ significantly improves the fit to $\chi^{2} /$ d.o.f. $=97 / 64$ (the probability of chance improvement is $\sim 10^{-14}$ ). The best fit is found for a photon index of $\sim 1.8$ and a cut-off energy at $\sim 80 \mathrm{keV}$. However, the model does not describe the spectrum below $15 \mathrm{keV}$ well, which requires a more complex description.

We then substituted this phenomenological model by a more physical thermal Comptonization model. The emission from the accretion shock onto the NS is well described by thermal Comptonization of the black body seed photons from the star (see Gierliński et al. 2002; Poutanen \& Gierliński 2003; Gierliński \& Poutanen 2005). We model the shock emission with the COMPPS model ${ }^{1}$ (Poutanen $\&$ Svensson 1996). In this model, the exact numerical solution of the Comptonization problem in different geometries is obtained by considering successive scattering orders. We approximate the accretion shock geometry by a plane-parallel slab at the NS surface. The main model parameters are the Thomson optical depth $\tau_{\mathrm{T}}$ across the slab, the electron temperature $k T_{\mathrm{e}}$, and the soft seed photon temperature $k T_{\text {seed. }}$. The emitted spectrum depends also on the angle between the normal and the line of sight $\theta$ which does not coincide with the inclination of system because of light bending. The seed photons are injected from the bottom of the slab. A fraction $\exp \left(-\tau_{\mathrm{T}} / \cos \theta\right)$ of these photons reaches the observer directly, while the remaining part is scattered in the hot gas. Thus, the total spectrum contains unscattered black body photons and a hard Comptonized tail. The best

\footnotetext{
${ }^{1}$ comprs is available on the WWW at ftp://ftp.astro.su.se/pub/juri/XSPEC/COMPPS
} 
Table 1. Best fit parameters for rev-46 and rev-52 data.

\begin{tabular}{lll}
\hline \hline Dataset & rev-46 & rev-52 \\
Models & COMPPS & cOMPPS+DISKBB \\
\hline$N_{\mathrm{H}}\left(10^{22} \mathrm{~cm}^{-2}\right)$ & $0.56(\mathrm{f})$ & $0.56_{-0.02}^{+0.01}$ \\
$k T_{\text {disk }}(\mathrm{keV})$ & - & $0.43_{-0.04}^{+0.04}$ \\
$R_{\text {in }} \sqrt{\cos i}(\mathrm{~km})$ & - & $13.4_{-1.3}^{+2.2}$ \\
$k T_{\mathrm{e}}(\mathrm{keV})$ & $18_{-3}^{+3}$ & $37_{-10}^{+28}$ \\
$k T_{\text {seed }}(\mathrm{keV})$ & $0.8_{-0.05}^{+0.04}$ & $0.75_{-0.04}^{+0.04}$ \\
$\tau_{\mathrm{T}}$ & $2.7_{-0.2}^{+0.1}$ & $1.7_{-0.8}^{+0.5}$ \\
$\left.A_{\text {seed }}(\mathrm{km})^{2}\right)$ & $86_{-8}^{+15}$ & $26_{-12}^{+23}$ \\
$\cos \theta$ & $0.80_{-0.09}^{+0.06}$ & $0.79_{-0.06}^{+0.07}$ \\
$\chi^{2} /$ d.o.f. & $36 / 62$ & $214 / 198$ \\
$L_{0.1-200 \mathrm{keV}}\left(10^{37} \mathrm{erg} \mathrm{s}^{-1}\right)$ & 1.26 & 0.36 \\
\hline
\end{tabular}

a Assuming a distance of $8 \mathrm{kpc}$.

fit with $\chi^{2} /$ d.o.f. $=36 / 62$ is then obtained for $k T_{\text {seed }}=0.8 \mathrm{keV}$, $k T_{\mathrm{e}} \approx 20 \mathrm{keV}, \tau_{\mathrm{T}}=2.7$, and $\cos \theta=0.8$ (see Table 1 ). The model also allows us to determine the apparent area of the seed photons, which turns out to be $A_{\text {seed }} \sim 86(D / 8 \mathrm{kpc})^{2} \mathrm{~km}^{2}$ in the best fit. This corresponds to a hot spot radius of $\sim 5 \mathrm{~km}$ in this outburst phase.

No additional thermal-like component (either blackbody or disk blackbody) was required by the fit, very likely because the bulk of its emission occurs below $3 \mathrm{keV}$, outside the covered energy range. Neither iron line emission nor a reflection component were significantly detected.

The best fit parameters of the model together with its errors are reported in Table 1 . The unabsorbed $E F_{\mathrm{E}}$ spectrum and the residuals of the data to the model are shown in Fig. 4.

\subsubsection{The rev-52 spectrum}

The INTEGRAL rev-52 dataset was obtained 22 days later than rev-46, this time also simultaneously with the XMM-Newton ToO observation. We were therefore able to construct a combined spectrum covering the $0.5-200 \mathrm{keV}$ range. The thermal emission from the accretion disk can contribute at low energies in the XMM-Newton range. The presence of the emission from the accretion disk is supported by the decrease of the pulse fraction below $2 \mathrm{keV}$ (Campana et al. 2003; Kirsch et al. 2004). We model this emission with DISKBB, a multi-temperature disk model of Mitsuda et al. (1984). It is characterized by the inner disk temperature $k T_{\text {in }}$ and normalization which can be transformed to the inner disk radius $R_{\text {in }}$. The spectrum was then fitted with this component combined with a simple PL, everything photoelectrically absorbed. The model gives a good fit with $\chi^{2} /$ d.o.f. $=237 / 200$. The absorption is determined to be $N_{\mathrm{H}}=0.56 \times 10^{22} \mathrm{~cm}^{-2}$, comparable to that found by Campana et al. (2003), and the best PL index is $\Gamma \sim 1.9$. However, the inner disk radius, $R_{\text {in }} \sqrt{\cos i}$, is then around $2 \mathrm{~km}$, which is not consistent with the disk size, assuming a $10 \mathrm{~km} \mathrm{NS}$ radius. To have a direct comparison with the results of the rev-46 data we

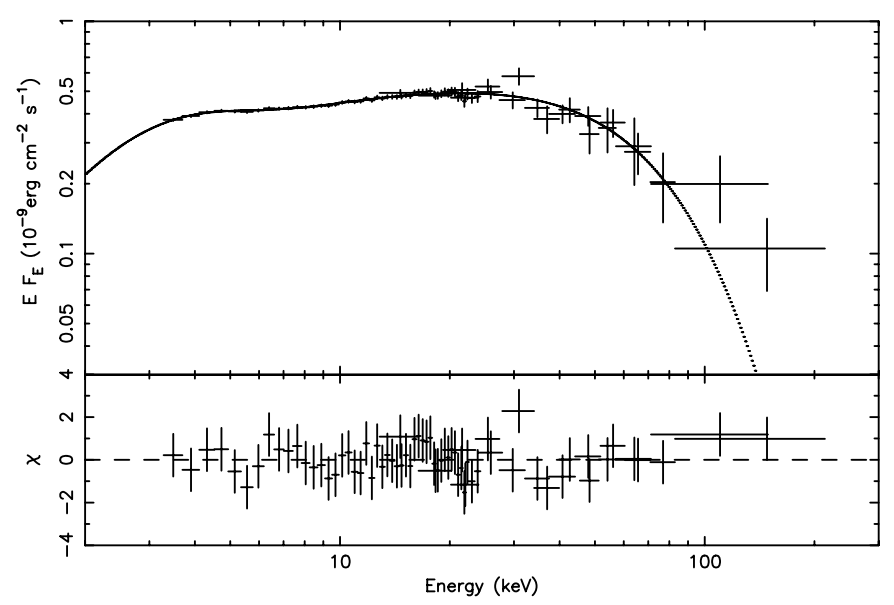

Fig. 4. The unfolded spectrum of XTE J1807-294 observed by INTEGRAL/ISGRI and RXTE/PCA/HEXTE during rev-46 along with the best fit COMPPS model. The lower panel shows the residuals between the data and the model.

replaced the PL component with COMPPS in a slab geometry assuming a black body seed photon spectrum.

The results of the DISKBB + COMPPS fit are reported in Table 1, while the $E F_{\mathrm{E}}$ spectrum and the residuals are shown in Fig. 5. With a typical plasma temperature of $k T_{\mathrm{e}} \sim 40 \mathrm{keV}$, the joint INTEGRAL/XMM-Newton/RXTE fit is at variance with the parameters derived from the low energy XMM-Newton data only which gave $k T_{\mathrm{e}} \sim 10 \mathrm{keV}$ (see Campana et al. 2003), confirming the importance of high energy observations. Comparison with the rev-46 results shows that the decay of the outburst is marked by a constant seed photon temperature, a significant increase of plasma temperatures correlated with a significant decrease of the scattering opacity. The fit required a inner disk radius, $R_{\text {in }} \sqrt{\cos i} \sim 13 \mathrm{~km}$, this time fully compatible with the radius of a NS. The apparent area of the seed photons is $A_{\text {seed }} \sim 26(D / 8 \mathrm{kpc})^{2} \mathrm{~km}^{2}$, which is also what expected from a hot spot on a NS surface. The hard component contributes 83 per cent of the unabsorbed flux in the $0.1-200 \mathrm{keV}$ energy range. Also in this case reflection was not significantly detected.

\section{Companion star and the system geometry}

The orbital elements of XTE J1807-294 have been derived from a re-analysis of the XMM-Newton temporal data, using a Doppler pulse-variation procedure instead of the complex 2D-parameter search made by Campana et al. (2003) and Kirsch et al. (2004). The barycentric photon arrival times were grouped into $240 \mathrm{~s}$ intervals and the best period was determined using the $Z$-statistic (Buccheri et al. 1983). The resulting 39 best period values clearly show a sinusoidal modulation expected for a circular orbit (see Fig. 6). A sine-fit to the modulation yields a mean pulsation period of $0.005245948(4) \mathrm{s}$, a projected orbital radius of $a_{\mathrm{x}} \sin i=4.75(39) \times 10^{-3} \mathrm{lt}-\mathrm{s}$ and a barycentric positive-crossing time (mean longitude $+90^{\circ}$ ) of $T_{0}=2452721.168(2) \mathrm{JD}$, where the errors are given in parentheses. The poorly constrained orbital period was assumed here to be 40.0741 min (Markwardt et al. 2004). The spin period 


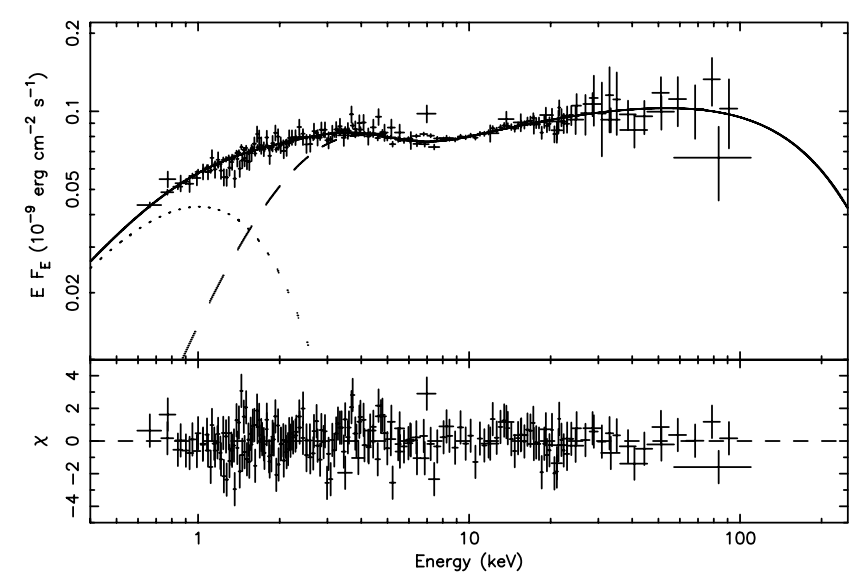

Fig. 5. Simultaneous INTEGRAL, XMM-Newton and RXTE spectra of XTE J1807-294 fitted with an absorbed disk black body, DISKBB, plus COMPPS model. The EPIC-pn and MOS2 spectra in the $0.5-10 \mathrm{keV}$ range and PCA/HEXTE in the $3-200 \mathrm{keV}$ and IBIS/ISGRI in the 20-200 keV range are shown. The DISKBB model is shown by a dotted curve, the dashed curve gives the coMPPS model, while the total spectrum is shown by a solid curve. The lower panel presents the residuals.

and orbit projected radius values are consistent with the previous determinations by Campana et al. (2003) (once corrected for typing errors) and Kirsch et al. (2004), but with more reliable uncertainties. However, the epoch of mean longitude differs very significantly, by more than $0.005 \mathrm{~d}$, most probably due to the inaccuracy of the complex method used by these authors. The introduction of an eccentricity does not improve the fit. A small linear slope is visible in the residuals (see Fig. 6), but not significant. However phase deviations (rms of $\sim 15 \mathrm{~ns}$ ) are also seen (Fig. 6), corresponding to highly erratic period variations. Such a behavior was already noted by Markwardt (2004) who reported apparent spin-up and spindown rate of $\sim \pm 3 \times 10^{-16} \mathrm{~s} \mathrm{~s}^{-1}$ on longer timescales. The instability of the period may therefore be also present on very short timescale ( $\sim 5 \mathrm{~min})$ which is a strong indication that we do not see the real clock, but rather a moving spot at the surface of the neutron star.

With a mass function of $f(m)=1.49_{-0.34}^{+0.40} \times 10^{-7} M_{\odot}$, the minimum companion mass obtained for inclination of $i=90^{\circ}$ is $M_{\mathrm{c}}=0.0053,0.0066$ and $0.0084 M_{\odot}$ for $M_{\mathrm{x}}=1.0,1.4$ and $2.0 M_{\odot}$, respectively.

The assumption of a Roche lobe-filling companion (Paczyński 1971) implies the mass-radius relation shown in Fig. 7 (thick line). In the very low-mass regime, recent models of low-mass degenerate dwarfs have been produced incorporating the effect of different compositions and temperatures (Deloye \& Bildsten 2003). The corresponding $M_{\mathrm{c}}$ versus $R_{\mathrm{c}}$ relations are also shown in Fig. 7. For a mean inclination of $i \sim$ $60^{\circ}$, only low temperature $\mathrm{C}$ dwarfs and $\mathrm{O}$ dwarfs are allowed, clearly excluding He dwarfs which would require very low inclinations $i \leq 30^{\circ}$. This strongly constrains the evolution of the system.

An interesting constraint is given by the minimum accretion rate driven by gravitational radiation in a close binary (see Verbunt \& van der Heuvel 1995, for a review). The outburst

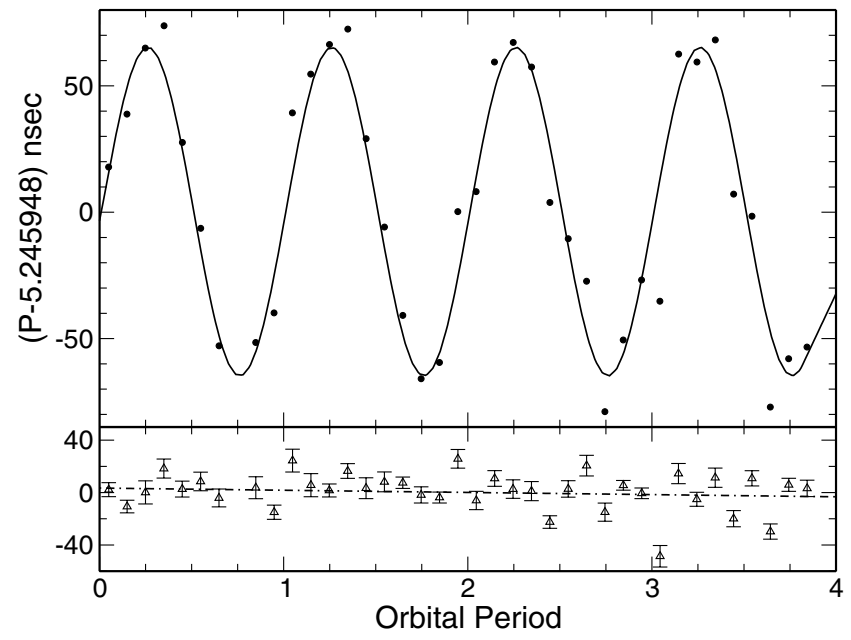

Fig. 6. Doppler variations of the spin period as a function of orbital phase and the best sine fit. Each point corresponds to a $240 \mathrm{~s}$ interval. Significant residuals are seen with rms of $\sim 15 \mathrm{~ns}$.

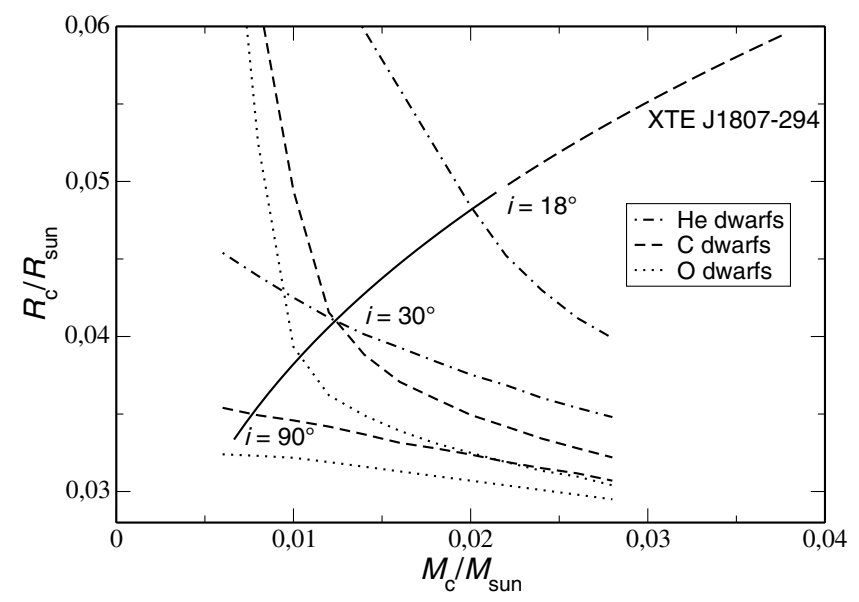

Fig. 7. Companion mass $M_{\mathrm{c}}$ vs. radius $R_{\mathrm{c}}$ plane, showing the Roche lobe constraints for XTE J1807-294 (thick line), for $M_{\mathrm{x}}=1.4 M_{\odot}$. The figure shows also the low mass regime degenerate dwarf models incorporating different compositions (dotted $\mathrm{O}$, dashed $\mathrm{C}$, dotdash $\mathrm{He})$ and low $\left(10^{4} \mathrm{~K}\right)$ or high $\left(3 \times 10^{6} \mathrm{~K}\right)$ central temperatures (lower and upper curves).

main peak was observed on February 21, 2003 (52 691 MJD), and follows the outburst shape (see Fig. 2); we estimated the total flux to be $7.7 \times 10^{-3} \mathrm{erg} \mathrm{cm}^{-2}$. Following Bildsten \& Chakrabarty (2001) we can constrain the source distance as a function of inclination angle and companion mass through the mass function and mass loss rate due to gravitational radiation. From randomly selected orbits, the probability of having an inclination $i<i_{\mathrm{o}}$ is $1-\cos i_{\mathrm{o}}$, so that a maximum mass at a confidence level $95 \%$ can be derived for $i<18^{\circ}$ with the value $M_{\mathrm{c}} \leq 0.022 M_{\odot}$. Note that for this source the recurrence time of the outburst is unknown. Assuming a recurrence time of 5 years, the source is calculated to be at $8 \mathrm{kpc}$ with an inclination angle of $18^{\circ}\left(M_{\mathrm{c}}=0.022 M_{\odot}\right)$. At a mean inclination angle of $60^{\circ}\left(M_{\mathrm{c}}=0.0077 M_{\odot}\right)$, the source must be located at $3 \mathrm{kpc}$. With a recurrence time of 39 years, the source is located at $8 \mathrm{kpc}$ with an inclination angle of $60^{\circ}$. This calculation predicts that a He dwarf companion star is favorable for a low 
inclination angle and the system located at $\sim 8 \mathrm{kpc}$. A high inclination implies that the system be located at $\sim 3 \mathrm{kpc}$ with a $\mathrm{C} / \mathrm{O}$ dwarf companion star. A solution of this problem can be obtained if a future outburst is observed, giving the recurrence time of outburst.

The mass transfer driven by gravitational radiation implies an inclination $i>60^{\circ}$, assuming a source distance of $8 \mathrm{kpc}$ and NS masses between 1.0 and $2.0 M_{\odot}$, giving only a long recurrence time of the outburst. On the other hand, there were no X-ray eclipses or dips detected in the EPIC-pn light curves. We therefore put an upper limit on the binary inclination of $i<$ $83^{\circ}$ for a Roche lobe-filling companion. A very low inclination is also possible, since that would imply a high companion mass and thus a large mass transfer rate. There is also no evidence for an X-ray modulation during the binary period, which might have implied propagation through a scattering atmosphere in a near edge-on geometry (Bildsten \& Chakrabarty 2001). If the source is most likely at an inclination of $60^{\circ}-83^{\circ}$, we get the inner disk radius (see Table 1 ) in the range $20-40 \mathrm{~km}$.

\section{Conclusions}

We have found that the spectrum of XTE J1807-294 is well described by a combination of thermal Comptonization and a disk black body. The electron temperature of $k T_{\mathrm{e}} \sim 40 \mathrm{keV}$ is considerably larger than previously determined from lower energy observations (Campana et al. 2003). The hard spectral component contributes most of the observed flux (83 percent), even though a soft component (disk black body) is required by the data. If the accretion disk is truncated at the magnetosphere radius, where the material can accrete along the magnetic field towards the pole of a NS, the matter over the pole is heated by a shock to a temperature of $\sim 40 \mathrm{keV}$. The hot spot at the surface with temperature $\sim 0.8 \mathrm{keV}$ gives rise to the seed photons for Comptonization in the hot plasma. Since the hard X-ray emission is pulsed (see e.g. Gierliński \& Poutanen 2005), a fraction of it must originate from the regions confined by the magnetic field. The most obvious source of hard X-rays is the place where material collimated by the magnetic fields impacts the surface.

If we consider the inclination of the system to be $60^{\circ}<i<$ $83^{\circ}$, this allows us to determine the inner disk radius which lies in the range $20-40 \mathrm{~km}$ (for the distance of $8 \mathrm{kpc}$ ). Many characteristics of the source are similar to those observed in other MSPs, SAX J1808.4-3658 (Gierliński et al. 2002; Poutanen \& Gierliński 2003) and XTE J1751-305 (Gierliński \& Poutanen 2005).

Acknowledgements. M.F. and A.M. acknowledge CNES and the CNRS "GdR Phénomènes Cosmiques de Haute Énergie” for financial support. JP is supported by the Academy of Finland and the NORDITA Nordic project on High Energy Astrophysics.

\section{References}

Arnaud, K. A. 1996, in Astronomical Data Analysis Software and Systems V, ed. G. H. Jacoby, \& J. Barnes (San Francisco: ASP), ASP Conf. Ser., 10117

Bhattacharya, D., \& van den Heuvel, E. P. J. 1991, Phys. Rep., 203, 1 Bildsten, L., \& Chakrabarty, D. 2001, ApJ, 557, 292

Buccheri, R., Bennett, K., Bignami, G. F., et al. 1983, A\&A, 128, 245

Campana, S., Ravasio, M., Israel, G. L., et al. 2003, ApJ, 954, L39

Courvoisier, T. J.-L., Walter, R., Beckmann, V., et al. 2003, A\&A, 411, L57

Deloye, C. J., \& Bildsten, L. 2003, ApJ, 598, 1217

Eckert, D., Walter, R., Kretschmar, P., et al. 2004, Astr. Tel., 352

Finger, M. H., Falanga, M., Markwardt, C. B., \& Wilson, C. A. 2005, ApJ, submitted

Gierliński, M., \& Poutanen, J. 2005, MNRAS, submitted [arXiv: astro-ph/0411716]

Gierliński, M., Done, C., \& Barret, D. 2002, MNRAS, 331, 141

Goldwurm, A., David, P., Foschini, L., et al. 2003, A\&A, 411, L223

Gros, A., Goldwurm, A., Cadolle-Bel, M., et al. 2003, A\&A, 411, L179

Jansen, A., Lumb, D., Altieri, B., et al. 2001, A\&A, 365, L1

Kirsch, M. G. F., Mukerjee, K., Breitfellner, M. G., et al. 2004, A\&A, 423, L9

Lebrun, F., Leray, J.-P., Lavocate, Ph., et al. 2003, A\&A, 411, L141

Lund, N., Budtz-Joergensen, C., Westgaard, N. J., et al. 2003, A\&A, 411, L231

Markwardt, C. B. 2004, in X-Ray Timing 2003: Rossi and Beyond, ed. P. Kaaret, F. K. Lamb, \& J. H. Swank (Melville, NY: AIP), 209

Markwardt, C. B., Swank, J. H., Strohmayer, T. E., in 't Zand, J. J. M., \& Marshall, F. E. 2002, ApJ, 575, L21

Markwardt, C. B., \& Swank, J. H. 2003, IAU Circ., 8144

Markwardt, C. B., Juda, M., \& Swank, J. H. 2003, IAU Circ., 8095

Markwardt, C. B., Swank, J. H., \& Strohmayer, T. E. 2004, Astr. Tel., 353

Mitsuda, K., Inoue, H., Koyama, K., et al. 1984, PASJ, 36, 741

Poutanen, J., \& Gierliński, M. 2003, MNRAS, 343, 1301

Poutanen, J., \& Svensson, R. 1996, ApJ, 470, 249

Paczyński, B. 1971, ARA\&A, 9, 183

Strohmayer, T. E. 2001, Adv. Space Res., 28, 511

Strüder, L., Briel, U., Dennerl, K., et al. 2001, A\&A, 365, L18

Turner, M. J. L., Abbey, A., Arnaud, M., et al. 2001, A\&A, 365, L27

Ubertini, P., Lebrun, F., Di Cocco, G., et al. 2003, A\&A, 411, L131

van der Klis, M. 2000, ARA\&A, 38, 717

Verbunt, F., \& van den Heuvel, E. P. J. 1995, in X-ray binaries, ed. W. H. G. Lewin, J. van Paradijs, \& E. P. J. van den Heuvel (Cambridge: Cambridge University Press), 457

Winkler, C., Courvoisier, T. J.-L., Di Cocco, G., et al. 2003, A\&A, 411, L1

Wijnands, R., \& van der Klis, M. 1998, Nature, 394, 344

Wijnands, R. 2005, in Pulsars New Research (New York: Nova Science Publishers), in press [arXiv: astro-ph/0501264]

Zhang, F., Qu, J. L., LI, X. D., \& Falanga, M. 2005, ApJ, submitted 